Bonn ergeben sich z. B. bei der $p_{\mathrm{H}}$-Berechnung nach Ellenberg z. Tl. so erhebliche Abweichungen von den tatsächlichen Messungen, daß die Ergebnisse wertlos werden, wie die Zusammenstellung von ganz zufällig herausgegriffenen Beispielen von $p_{\mathrm{H}^{-}}$Zahlen ( $\mathrm{N}$ a u m a n n 1951) zeigt:

$\begin{array}{lrrrrr}\text { Aufnahme } & 173 & 124 & 122 & 297 & 125 \\ p_{\mathrm{H}} \text { gemessen } & 5,1 & 6,3 & 6,5 & 4,2 & 8,2 \\ p_{\mathrm{H}} \text { berechnet } & 5,2 & 4,4 & 5,2 & 6,3 & 6,8 \\ & 5,8 & 5,3 & 5,9 & 6,9 & 7,2\end{array}$

(Die Unterschiede in der Berechnung ergeben sich durch den von E. angegebenen Schwankungsbereich seiner Reaktionszahlen.) Auch wenn man die regionale Gültigkeit der Methode kennt, so ist über die Ausdehnung dieses Gültigkeitsbereiches noch nichts bekannt.

Diese Nachteile werden vermieden, wenn man Trennarten (Differentialarten) der Untergesellschaften (Subass., Varianten, Subvar.) innerhalb ihrer Assoziation als Zeiger wertet, d. h. statt einzelner Arten oder deren Gruppen diese Gesellschafts-Einheiten eicht und den untersuchten Einzelbestand dazu in Beziehung setzt. Schon 1939 hatte E. übrigens erkannt, daß die gleiche Art in verschiedenen Gesellschaften, d. h. innerhalb verschiedener soziologischer Wirkungsgefüge und auf verschiedenen Standorten bestimmten Faktoren gegenüber abweichenden Zeigerwert besitzt.

Diese grundsätzlichen Bemerkungen können den Wert der zahlreichen überaus anregenden Einzelbeobachtungen des Buches in keiner Weise beeinträchtigen. Wir glauben freilich, daß die reichen ökologischen Erfahrungen des Verfassers und seine ausgesprochene Begabung der Darstellung bei festerer soziologischer Unterbauung Ergebnisse von allgemeinerer Gültigkeit erzielen würden. Der 2. Band seiner "Landwirtschaftlichen Pflanzensoziologie“ scheint uns diese Auffassung zu bestätigen.

R. T ü x e n, Stolzenau (W.).

Die Schmetterlinge Mitteleuropas. Von W. F o r s t e $r$ und Th. A. W o h lf a h r t. Franckh'sche Verlagshandlung Stuttgart. Bd. I/II (ca. 256 und 128 S. mit rd. 500 vielfarbigen Bildern auf 27 Tafeln). 6 Lieferungen zu je DM 10.-.
Eine Würdigung des Werkes, von dem soeben die erste Lieferung erschien, bringen wir nach Vorliegen der Bände I (Allg. Teil) und II (Tagfalter).

Vier Vorträge von Günther Just. Mit einem Geleitwort von E d u a r d S p r a n g e r. Springer-Verlag, Berlin, Göttingen, Heidelberg 1951. 64 S. mit 1 Porträt; Preis DM 4.80.

Im ersten Vortrag, „Die Stellung des Menschen im Reiche des Lebendigen“, werden in lapider Kürze zentrale Aufgaben der Anthropologie an Beispielen veranschaulicht und in ihrer Verknüpfung mit anderen Disziplinen, der Tierpsychologie, der Genetik, Konstitutionswissenschaft und Psychiatrie aufgezeigt. Justs umfassende Bildung ließ ihn anthropologische Probleme selbst in Bereichen der Literaturwissenschaft sehen.

Systematisch werden die „Gegenwartsprobleme der Anthropologie“ im dritten, zeitlich ein Jahr früher liegenden Vortrage behandelt, der um seiner Verbindung mit dem ersten hier vorweggenommen sei; Just gruppiert um drei „Felder“: Erforschung der Stammesgeschichte des Menschen und der Formenmannigfaltigkeit der Menschenrassen; Konstitutions- und namentlich Erbbiologie; Sozialanthropologie und angewandte Anthropologie. Für Fernerstehende sei bemerkt, daß psychologische Aspekte für Just als Naturwissenschaftler in die genannten Disziplinen zwanglos einfügbar sind, vornehmlich bei der Konstitutionsbiologie. Sein Forschungseifer wandte sich dieser Disziplin, und zwar gerade ihrem psychologischen Sektor, bevorzugt zu. So steht der Vortrag „Über die Beurteilung geistiger Leistung" mit Recht im Mittelpunkt des kleinen Buches. Das „Problem der Elite“, Gegenstand eines im Vorjahr erschienenen Buches eines großen deutschen Physikers, angeschnitten auch in Erns t J ü n g r s soeben erschienem Werke "Der Waldgang“, wird hier (wie in dem letzten Vortrage "Alte und neue Sozialanthropologie") in einer so würdigen, tiefernsten und dabei wegweisenden Form behandelt, daß man das Buch in die Hände aller im öffentlichen Leben Stehender wünscht.

M üll e r - U s ing, Hann.-Münden.

\title{
NACHRICHTEN
}

\section{Tagung der Gesellschaft Deutscher Naturforscher und Ärzte}

vom 21. bis 25. September 1952 in Essen

Aus dem Programm:

Sonntag, 21. September

Eröffnungssitzung, Festrede des 1. Vorsitzenden, Prof.

A. B u t e $\mathrm{n}$ a $\mathrm{nd} \mathrm{t}$, Tübingen.

Montag, 22. September

50 Jahre Genetik. Vorträge von Prof. A. K ü h n, Tü-

bingen, Prof. H. M a r q u a r d t, Freiburg i. Br., Prof.

F. O e hlkers, Freiburg i. Br., Prof. E. H a g e -

d orn, Zürich, Prof. A. B u te n a n d t, Tübingen.

Dienstag, 23. September

50 Jahre Virusforschung. Vorträge von Dr. W e i d e l, Tübingen, Dr. S c h r a m m, Tübingen, Prof. T. C a s - pers s on, Stockholm, Prof. W. Kikuth, Düsseldorf.

Mittwoch, 24. September

Elektrobiologie. Vorträge von Prof. H. S c hä f e r, Heidelberg, Prof. A. E. K orn müller, Göttingen, Prof. H. L u ll i es, Saarbrücken, Prof. H. A u trum, Göttingen.

Geologie. Vorträge von Prof. K. O b e r s t e - B r in k, Aachen, Prof. R. P ot o n i é, Krefeld.

Schlußsitzung. Prof. G. R. v. K o e n i g s w a $1 \mathrm{~d}$, Utrecht (Phylogenie des Menschen).

Anfragen sind zu richten an die Geschäftsstelle der Gesellschaft Deutscher Naturforscher und Ärzte in Wuppertal-Elberfeld, Friedrich-Ebert-Straße 217. 